\title{
Multiple Layer Symbol Design for Use with Internet Maps
}

\author{
Rex G. Cammack ${ }^{\mathrm{a}^{*}}$, Paul Hunt ${ }^{\mathrm{a}}$ \\ ${ }^{a}$ University of Nebraska Omaha,rcammack@unomaha.edu;phunt@unomaha.edu \\ * Corresponding author
}

Keywords: Internet maps, Near real-time mapping, Layered Symbols, Symbology

\begin{abstract}
:
Mapping both qualitative and quantitative point data has been a mainstay in cartography. Research on symbolization of point data has branched across several areas of study: Geometric, Pictorial, Icons, Point Clustering and other topics. In this research, an examination of symbol design and representation on internet maps is the focus. For this project, the data being presented are near real-time dataset. The Internet provides access to many near real-time dataset but research on effectively presenting this data is only just beginning. This project is broken into to parts. Frist, the system implementation design and deployment. The second part is the symbol design and representation model.
\end{abstract}

For system design we have approached this from a web data scraping and serverless environment approach. The near real-time data is gathered and appended to existing datasets using web scraping methods. One part of this project is that the near real-time data has to be coupled with historical data. On the map end of the project, map viewers can look at historical animations up to the present or see a near real-time surveillance type of map. This system design leverages cloud based serverless technology and data storage approaches. Some server-based technologies, like Web Mapping Services (WMS) are used to deploy the data to increase it usability.

Symbol representation of data is the second part of this research project. We examined a brute force approach for representation where symbols were designed in every possible combination. The number of symbol combinations is manageable for a single classified data dimension. When a cartographer wants to combine multiple values to form the map symbol, a symbol design algorithm needs to be implemented. This type of symbol design comes from the ideas underlaying the work on multivariate mapping of local areas using Chernoff faces. In this project we use more abstract/geometric types of symbols but it should be noted that even Chernoff faces symbology can be used. During the project we examined the number of mapping dimensions and found that a clear symbol model made increasing mapping dimension simpler to implement. This project is intended as a first step and that map use testing needs to be conducted for better understanding of the strength and limitation of this symbology method. To make the symbol model simpler we split the symbol into layers. With the symbol in layered format, we can attach a mapping dimension to one of the symbol layers. Two methods of layering symbols were examined. The first method being symbol overlaying. Symbol overlaying simply means that each part of the symbol is created by drawing different layers in the same location. With careful design of the symbol model this method worked. But with complex symbol models, the devolution of the symbol into multiple layers is far from ideal. Next, we turned to a multiple layer single symbol design. With the single symbol model, symbol layers represent multiple values and still have a composite design. This approach does create a more complex symbol model when working with web maps, but it is a more manageable final implementation. This methods strength is best noticed as the number of dimensions on the symbol increases.

In conclusion this research demonstrates that complex multi-value datasets in near real-time can be presented cartographically. Layered symbol models can be used to represent multiple variables in a dynamic manner for Internet maps. Research still needs to be done on how to represent this type of complex symbology via a legend so map readers understand the nature of the presentation. Additionally, Map use and usability research should examine the merit of this layered symbol method. 